\title{
Global hybrid-gyrokinetic simulations of fast-particle effects on Alfvén Eigenmodes in stellarators
}

\author{
A. Mishchenko* and A. Könies \\ Max Planck Institute for Plasma Physics, \\ Wendelsteinstr. 1, 17491 Greifswald, Germany \\ T. Fehér \\ Max Planck Institute for Plasma Physics, \\ Boltzmannstr. 2, 85748 Garching, Germany \\ R. Kleiber, M. Borchardt, and J. Riemann \\ Max Planck Institute for Plasma Physics, \\ Wendelsteinstr. 1, 17491 Greifswald, Germany \\ R. Hatzky \\ Max Planck Institute for Plasma Physics, \\ Boltzmannstr. 2, 85748 Garching, Germany \\ J. Geiger and Yu. Turkin \\ Max Planck Institute for Plasma Physics, \\ Wendelsteinstr. 1, 17491 Greifswald, Germany
}

(Dated: March 5, 2014)

* alexey.mishchenko@ipp.mpg.de 


\begin{abstract}
Simulations of gyrokinetic energetic ions interacting with the magneto-hydrodynamic (MHD) Alfvén Eigenmodes are presented. The effect of the finite fast-ion orbit width and the finite fast-ion gyroradius, the role of the equilibrium radial electric field, as well as the effect of anisotropic fastparticle distribution functions (loss-cone and ICRH-type distributions), are studied in Wendelstein 7-X stellarator geometry using a combination of gyrokinetic particle-in-cell and reduced MHD eigenvalue codes. A preliminary stability analysis of a HELIAS reactor configuration is undertaken.
\end{abstract}




\section{INTRODUCTION}

Alfvén instabilities caused by energetic ions have been seen in many fusion experiments, both in tokamak [1] and stellarator/heliotron [2,3] devices. The associated physics has extensively been studied theoretically in tokamak geometry [4-6]. In comparison to tokamaks, there are many similarities in the properties of Alfvénic waves and fast particles in stellarator geometry. There are, however, also discrepancies, which can be caused by the difference in the magnetic shear, the lack of axisymmetry, the absence or smallness of a net toroidal plasma current, more complicated particle orbits in stellarators etc. [7]. The theoretical study of fast-particle destabilized Alfvénic instabilities in 3D geometry was pioneered by Kolesnichenko from 2001 onwards [8, 9]. At that time, the physics related to the fast-particle transport induced by the Alfvén waves in tokamak plasmas had already been studied intensively. The interest in fast-ion-driven Alfvénic instabilities in 3D has increased since the appearance of drift-optimized [10] stellarators, such as the Wendelstein 7-AS (W7-AS) [11], and large heliotrons such as the Large Helical Device (LHD) [12]. It has become apparent that Alfvén modes may play an important role in burning stellarator plasmas (e. g. in a HELIAS reactor $[13,14]$ ). Recent interest has been motivated not only by projections to stellarator reactor-relevant conditions but also by experimental findings of unstable Toroidal Alfvén Eigenmodes (TAE) and Global Alfvén Eigenmodes (GAE) in LHD [2] and in W7-AS [3]. The basic structure of the shear Alfvén spectrum in 3D and the most important stellarator-specific global modes (such as the Mirror Alfvén Eigenmodes or Helical Alfvén Eigenmodes) were discussed in Ref. [8]. Reference [9] was dedicated to a study of the relevant wave-particle resonances using a hybrid approach: Alfvén Eigenmodes were computed in a fluid approximation; for the kinetic fast ions, the power-transfer integral was evaluated analytically. The fast ions were treated in the zero-orbit-width approximation, and the effect of trapped ions was neglected. A similar study has been undertaken for LHD geometry in Ref. [15], and the effect of localized energetic ions on the Alfvénic stability in optimized stellarators has been considered in Ref. [16].

Numerically, a number of tools have been developed to study fast-particle instabilities in stellarators (see a comprehensive review in Ref. [7]). Most of these tools use a hybrid fluid-kinetic approach. In this paper, we employ a hybrid version of the EUTERPE code [17] to study the fast-particle destabilization of Alfvén Eigenmodes in stellarator plasmas. 
In this approach [18], the Alfvén Eigenmodes are computed using the eigenvalue solver CKA $[18,19]$ (employing the ideal-MHD version of Ohm's law). The resulting eigenmode structure and frequency are used when following the particles in the framework of the gyrokinetic particle-in-cell code EUTERPE [17]. The power transfer can be evaluated during this procedure, which is then used to determine the linear growth rate of the mode [18]. Details and benchmarks of the method will be published elsewhere. In this approach, we can naturally include all the effects associated with the finite orbit width and the finite Larmor radius of the fast ions. All kinds of trapped particles (helically-trapped, toroidally-trapped, transitioning etc.) are consistently included in the description as well as the effect of the equilibrium radial electric field. The real magnetic geometry (computed numerically with the VMEC code [20]) is accounted for. One can choose arbitrary fast-particle background distribution functions: a conventional Maxwellian, slowing-down, beam-like, loss-cone, or more complicated (e. g. realistic-NBI) distribution functions. We study the aforementioned effects in context of Alfvén Eigenmode stability for the drift-optimized quasi-omnigeneous configuration W7-X [21, 22]. Also, a preliminary analysis of the Alfvénic stability is carried out for a stellarator reactor (HELIAS [14]) configuration.

The structure of our paper is as follows. In Sec. II, the basic equations and their numerical treatment are discussed. The simulations are presented in Sec. III. Our conclusions are summarised in Sec. IV.

\section{BASIC EQUATIONS AND NUMERICAL APPROACH}

We use the linearised version of the three-dimensional $\delta f$ PIC-code EUTERPE [17]. The code is electromagnetic and can treat all particle species (ions, electrons, energetic particles, impurities etc.) kinetically. In the hybrid approach [18], we solve the gyrokinetic equation for the fast ions employing the $p_{\|}$-formulation (see Ref. [23] for details). The fastparticle distribution function is split into a background part and a small time-dependent perturbation, $f=F_{0}+\delta f$. The background distribution function can be chosen freely. In the following, we consider a number of background distribution functions, both isotropic and anisotropic ones (see Sec. III for details).

If the amplitude of the field perturbation is assumed to be small $\left(\delta f_{s} / F_{0 s} \ll 1\right)$, the 
first-order perturbed distribution function is found from the linearized Vlasov equation:

$$
\frac{\partial \delta f}{\partial t}+\dot{\mathbf{R}}^{(0)} \cdot \frac{\partial \delta f}{\partial \mathbf{R}}+\dot{v}_{\|}^{(0)} \frac{\partial \delta f}{\partial v_{\|}}=-\dot{\mathbf{R}}^{(1)} \cdot \frac{\partial F_{0}}{\partial \mathbf{R}}-\dot{v}_{\|}^{(1)} \frac{\partial F_{0}}{\partial v_{\|}} .
$$

Here, $\left[\dot{\mathbf{R}}^{(0)}, \dot{v}_{\|}^{(0)}\right]$ correspond to the unperturbed gyro-centre position and parallel velocity, and $\left[\dot{\mathbf{R}}^{(1)}, \dot{v}_{\|}^{(1)}\right]$ are the perturbations of the particle trajectories proportional to the electromagnetic field fluctuations [shown in Eqs. (3)-(6) below]. The perturbed part of the distribution function is discretized with markers:

$$
\delta f\left(\mathbf{R}, v_{\|}, \mu, t\right)=\sum_{\nu=1}^{N_{p}} w_{\nu}(t) \delta\left(\mathbf{R}-\mathbf{R}_{\nu}\right) \delta\left(v_{\|}-v_{\| \nu}\right) \delta\left(\mu-\mu_{\nu}\right),
$$

where $N_{p}$ is the number of markers, $\left(\mathbf{R}_{\nu}, v_{\| \nu}, \mu_{\nu}\right)$ are the marker phase space coordinates and $w_{\nu}$ is the weight of a marker. The equations of motion are

$$
\begin{aligned}
\dot{\mathbf{R}}^{(0)} & =v_{\|} \mathbf{b}^{*}+\frac{1}{q B_{\|}^{*}} \mathbf{b} \times\left(\mu \nabla B+q \nabla \Phi_{0}\right) \\
\dot{\mathbf{R}}^{(1)} & =-\frac{q}{m}\left\langle A_{\|}\right\rangle \mathbf{b}^{*}+\frac{1}{B_{\|}^{*}} \mathbf{b} \times\left(\nabla\langle\phi\rangle-v_{\|} \nabla\left\langle A_{\|}\right\rangle\right) \\
\dot{v}_{\|}^{(0)} & =-\frac{1}{m}\left(\mu \nabla B+q \nabla \Phi_{0}\right) \cdot \mathbf{b}^{*} \\
\dot{v}_{\|}^{(1)} & =-\frac{q}{m}\left(\nabla\langle\phi\rangle-v_{\|} \nabla\left\langle A_{\|}\right\rangle\right) \cdot \mathbf{b}^{*}
\end{aligned}
$$

with $\phi$ and $A_{\|}$being the perturbed electrostatic and magnetic potentials, $\mu$ the magnetic moment, $m$ the mass of the particle, $B_{\|}^{*}=\mathbf{b} \cdot \nabla \times \mathbf{A}^{*}, \mathbf{b}^{*}=\nabla \times \mathbf{A}^{*} / B_{\|}^{*}, \mathbf{A}^{*}=\mathbf{A}+$ $\left(m v_{\|} / q\right) \mathbf{b}$ the so-called modified vector potential, $\mathbf{A}$ the magnetic potential corresponding to the equilibrium magnetic field $\mathbf{B}=\nabla \times \mathbf{A}, \mathbf{b}=\mathbf{B} / B$ the unit vector in the direction of the equilibrium magnetic field, $q$ the charge of the energetic ion, and $\Phi_{0}$ the electrostatic potential corresponding to the background electric field (which is usually of neoclassical nature [24]). The gyro-averaged potentials are defined as usual:

$$
\langle\phi\rangle=\oint \frac{\mathrm{d} \theta}{2 \pi} \phi(\mathbf{R}+\boldsymbol{\rho}),\left\langle A_{\|}\right\rangle=\oint \frac{\mathrm{d} \theta}{2 \pi} A_{\|}(\mathbf{R}+\boldsymbol{\rho}),
$$

where $\boldsymbol{\rho}$ is the gyroradius of the particle and $\theta$ is the gyro-phase. Numerically, the gyroaverages are computed sampling a sufficient number of the gyro-points on the gyro-ring around the gyro-centre position of the marker [25, 26].

The perturbed electrostatic and magnetic potentials are found from the reduced idealMHD equations. The radial structure of the perturbed electrostatic potential and the frequency of the global mode are obtained by numerically solving the eigenmode problem (the 
Alfvén-wave equation) in 3D stellarator geometry:

$$
\begin{array}{r}
\omega^{2} \nabla \cdot\left(\frac{1}{v_{A}^{2}} \nabla_{\perp} \phi\right)+\nabla \cdot\left[\mathbf{b} \nabla_{\perp}^{2}(\mathbf{b} \cdot \nabla) \phi\right]+\nabla \cdot\left[\mathbf{b} \nabla \cdot\left(\frac{\mu_{0} j_{\|}}{B} \mathbf{b} \times \nabla \phi\right)\right]- \\
-\nabla \cdot\left(\frac{2 \mu_{0}}{B^{2}}[(\mathbf{b} \times \nabla \phi) \cdot \nabla p](\mathbf{b} \times \boldsymbol{\kappa})\right)=0
\end{array}
$$

Here, $v_{A}$ is the Alfvén velocity, $j_{\|}$is the ambient parallel current, $p$ is the background plasma pressure and $\boldsymbol{\kappa}=(\mathbf{b} \cdot \nabla) \mathbf{b}$ is the magnetic field-line curvature (note that the parallel-current and the magnetic-curvature corrections are usually small).

After the perturbed electrostatic potential $\phi$ has been obtained, the perturbed parallel magnetic potential $A_{\|}$can be solved from the parallel component of Ohm's law:

$$
E_{\|}=-\nabla_{\|} \phi-\frac{\partial A_{\|}}{\partial t}=0
$$

Numerically, the electrostatic and magnetic potentials are discretized with the finite-element method (Ritz-Galerkin scheme):

$$
\phi(\mathbf{x})=\sum_{l=1}^{N_{s}} \phi_{l} \Lambda_{l}(\mathbf{x}), \quad A_{\|}(\mathbf{x})=\sum_{l=1}^{N_{s}} a_{l} \Lambda_{l}(\mathbf{x}),
$$

where $\Lambda_{l}(\mathbf{x})$ are finite elements (tensor products of B-splines [27, 28]), $N_{s}$ is the total number of the finite elements, $\phi_{l}$ and $a_{l}$ are spline coefficients. Further details on the numerical approach can be found in Refs. [17-19].

The purpose of the simulations in this paper is to compute the linear growth rate of the eigenmode, which is given by the expression (see Ref. [18] for the derivation):

$$
\gamma=-\frac{1}{2 W_{\text {field }}} \frac{\mathrm{d} W_{\text {fast }}}{\mathrm{d} t}
$$

with the "field energy" defined as follows (neglecting here the small corrections related to the ambient pressure and the parallel current [18]):

$$
W_{\text {field }}=\frac{1}{2} \int \mathrm{d}^{3} x\left[\frac{m_{i} n_{0}}{B^{2}}\left(\nabla_{\perp} \phi\right)^{2}-\frac{1}{\mu_{0}}\left(\nabla_{\perp} A_{\|}\right)^{2}\right]
$$

and the "fast-particle power transfer" given by the expression:

$$
\frac{\mathrm{d} W_{\text {fast }}}{\mathrm{d} t}=-q_{\text {fast }} \int \mathrm{d}^{6} Z \delta f\left[\dot{\mathbf{R}}^{(0)} \cdot \nabla\left(\langle\phi\rangle-v_{\|}\left\langle A_{\|}\right\rangle\right)+\frac{1}{m}\left\langle A_{\|}\right\rangle \mathbf{b}^{*} \cdot\left(\mu \nabla B+q \nabla \Phi_{0}\right)\right]
$$

Here, $m_{i}$ is the bulk-ion mass, $n_{0}$ is the bulk-plasma density, $q_{\text {fast }}$ is the fast-particle charge, and $\delta f$ is the fast-particle distribution function. In our scheme, $W_{\text {field }}$ is precomputed on the 
grid when the simulation starts and the phase-space integral $\mathrm{d} W_{\text {fast }} / \mathrm{d} t$ must be computed on each time step using the markers. The electrostatic and magnetic potentials do not evolve

self-consistently. Instead, the expressions of the form $\hat{\Phi}_{\mathrm{AE}}(\mathbf{x}) \exp \left(i \omega_{\mathrm{AE}} t+\gamma t\right)$ are used for the fields with fixed fluid eigenfunction $\hat{\Phi}_{\mathrm{AE}}(\mathbf{x})$ and fixed fluid eigenfrequency $\omega_{\mathrm{AE}}$, both resulting from an MHD calculation.

Since the power transfer $\mathrm{d} W_{\text {fast }} / \mathrm{d} t$ is a function of time, we compute the growth rate of the eigenmode averaging Eq. (11) over a certain time interval. The average value is computed after the simulation has evolved for some time. This way we can avoid the issues associated with the initial noise and transient contributions to the wave-particle power transfer since the mean value of Eq. (11) converges to the actual growth rate of the eigenmode in the course of the simulation.

\section{SIMULATIONS}

\section{A. General description}

The simulations are performed in realistic stellarator magnetic geometry numerically calculated by the VMEC code [20]. The impact of Finite fast-ion drift-Orbit Width (FOW) effects and Finite fast-ion Larmor Radius (FLR) effects [which enter through the gyro-average of the perturbed field, see Eq. (7)] on the AE stability are considered as well as the role of the background (e. g. neoclassical) radial electric field and the background (unperturbed) fast-particle distribution function (e. g. anisotropy effects).

We consider a number of distribution functions, both isotropic and anisotropic ones. The simplest case is a conventional Maxwellian with constant temperature and a radially varying density (being the source of the free energy):

$$
F_{M}=n_{0}(s)\left(\frac{m}{2 \pi T_{0}}\right)^{3 / 2} \exp \left[-\frac{m v_{\|}^{2}}{2 T_{0}}\right] \exp \left[-\frac{m v_{\perp}^{2}}{2 T_{0}}\right]
$$

Here, $s$ is the normalised toroidal flux. The fast-ion density is as follows:

$$
n_{0}(s)=N_{0} \exp \left[-\kappa_{\mathrm{n}} \Delta_{\mathrm{n}} \tanh \left(\frac{s-s_{\mathrm{n}}}{\Delta_{\mathrm{n}}}\right)\right]
$$

The shape of the density profile can be tailored by adjusting the parameters $\Delta_{\mathrm{n}}$ (the width of the profile), $s_{\mathrm{n}}$ (position of the maximal density gradient), and $\kappa_{\mathrm{n}}$ (inverse density gradient 
length). In addition to the spatial gradients, the free energy source can be associated with an anisotropy of the velocity distribution function.

More realistic for fusion applications is the slowing-down distribution function given by

$$
F_{\mathrm{sd}}=\frac{S \tau_{s}}{4 \pi\left(v^{3}+v_{c}^{3}\right)} \Theta\left(v-v_{b}\right), \quad v_{c}=\left(\frac{n_{i} Z_{i}^{2}}{n_{e}} \frac{3 \pi^{1 / 2} m_{e}}{4 m_{i}}\right)^{1 / 3} v_{\text {the }}
$$

Here, $S$ is the fast-particle source (given usually by the rate of the fusion reaction), $\tau_{s}(s)$ is the slowing-down time, $v_{c}(s)$ is the "critical velocity" [determined by the electron temperature profile $T_{e}(s)$ ] above which the electron drag dominates over ion drag, and $\Theta(x)$ is the Heaviside function with $v_{b}$ the "birth" velocity.

While the fusion-born alpha particles are well described by the isotropic slowing-down distribution function, the fast ions generated by various heating methods (NBI or ICRH) are usually characterised by an anisotropic distribution function. We will consider a few examples of such distribution functions in Sec. III B.

\section{B. TAE mode in W7-X geometry}

We now consider W7-X geometry [21, 22]. The main parameters characterising the geometry are the rotational transform $\iota(s)$ shown in Fig. 1, the magnetic field at the axis $B_{0}=2.66 \mathrm{~T}$, the major radius $R_{0}=5.518 \mathrm{~m}$, the minor radius $r_{a}=0.496 \mathrm{~m}$ and the number of periods $N_{\text {per }}=5$ (reflecting the discrete symmetry of the stellarator). Both the bulk and fast ions are taken to be hydrogen. The bulk plasma density $n_{\text {bulk }}=10^{20} \mathrm{~m}^{-3}$ is assumed to be flat. The bulk plasma temperature $T_{i}=T_{e}=3 \mathrm{keV}$ is flat, too. The bulk-plasma beta corresponding to the resulting flat bulk-plasma pressure is $\beta_{\mathrm{bulk}}=2 \mu_{0} n_{\mathrm{bulk}}\left(T_{i}+T_{e}\right) / B_{0}^{2} \approx 0.034$. The density profile of the fast ions (which is the source of the free energy needed for the TAE destabilisation) is given by Eq. (15) with the parameters $N_{0}=10^{17} \mathrm{~m}^{-3}, \Delta_{\mathrm{n}}=0.2$, $s_{\mathrm{n}}=0.65$, and $\kappa_{\mathrm{n}}=3.0$. These parameters correspond (very roughly) to the NBI W7-X plasma. The magnetic field used corresponds to the high-mirror configuration. The resulting shear Alfvén continuum is shown in Fig. 2. The Fourier spectrum of the Alfvénic perturbations is

$$
\left[\phi, A_{\|}\right]=\sum_{m, n}\left[\phi_{m n}, A_{\| m n}\right] \exp (i m \theta+i n \zeta)
$$

with $\theta$ and $\zeta$ poloidal and toroidal angles, and $m$ and $n$ poloidal and toroidal mode numbers, respectively. Due to the symmetry of the magnetic field [the discrete symmetry with the 
period number $N_{\text {per }}=5$ and the stellarator symmetry $\left.(\theta, \zeta) \longrightarrow(-\theta,-\zeta)\right]$, this Fourier spectrum splits into $1+\left[N_{\text {per }} / 2\right]=3$ linearly independent mode families [29] (corresponding in W7-X e. g. to the toroidal mode numbers $n=0,1,2)$ where the geometry-related mode couplings are allowed only within a single mode family (e. g. the mode with $n=0$ couples to the modes with $n=5,10 \ldots$, but it does not interact linearly with the $n=1$ mode). The shear Alfvén continuum shown in Fig. 2 corresponds to $n= \pm 1$ mode family. One sees that a toroidicity-induced gap appears in the spectrum with a global eigenmode which has its frequency inside the gap. This is a TAE mode with the toroidal mode number $n=-6$ and dominant poloidal mode numbers $m=6$ and $m=7$. The radial eigenmode structure is shown in Fig. 3 [the effect of the ambient parallel current and plasma pressure has been neglected in this calculation, see Eq. (8)]. One sees that, indeed, the mode is global and has a characteristic TAE structure. The maximum of the mode is located, as usual in the TAE context, at the resonant position satisfying $k_{\| m}+k_{\| m+1}=0$ (with $m=6$ in the case shown here). Note that there are also other TAE modes in the gap.

The simplest model for the fast-ion distribution function is a Maxwellian. In this case, the velocity dependence of the distribution function (and the location of the wave-particle resonances) is determined by a single quantity: the fast-ion temperature $T_{f}$. The dependence of the mode growth rate on $T_{f}$ at fixed fast-ion density $N_{0}=10^{17} \mathrm{~m}^{-3}$ resulting from the hybrid-gyrokinetic simulations is shown in Fig. 4. First, one observes that the TAE mode can indeed be destabilised in Wendelstein 7-X geometry. Second, one can see the stabilising effects of finite orbit width (due to both the guiding-centre drifts and the gyro-motion of the fast ions). For this purpose, simulations including the fast-ion FLR [keeping the gyro-average in the equations of motion Eqs. (4) and (6)] are compared in Fig. 4 with the simulations where only the guiding-centre drifts of the fast ions have been included (no FLR). In both cases, the growth rate is bounded at high fast-ion temperatures. This effect is due to the finite width of the guiding-centre orbits of the ions. In addition, the growth rate in the case with the FLR effects included is smaller than in the case without the FLR effects (provided the fast-ion temperature is not too small) indicating that the fast-ion FLR effects (caused by their gyro-orbit) are stabilising with respect to the TAE mode, too. Such phenomena have also been observed in the tokamak context (see e. g. Ref. [30] and the references cited therein). In stellarators, the associated physics appears to be similar.

In Fig. 5, the growth rate of the same mode is plotted as the function of $T_{f}$ at fixed 
fast-ion beta $\beta_{f}(s=0.65)=0.003$ (for the bulk plasma, $\beta_{\text {bulk }} \approx 0.034$; it is a weak function of $s$ for the flat profiles considered here). Similarly to Fig. 4, the FOW and the FLR effects can be observed in Fig. 5. But, in contrast to Fig. 4, the mode growth rate decreases faster at high fast-ion temperatures (since the FOW/FLR stabilisation effect is not compensated by the increase in $\beta_{\text {fast }} \sim T_{\text {fast }}$ as is the case when the fast-ion density is fixed, see Fig. 4). Thus, the mode here is most destabilised at few hundred $\mathrm{keV}$ of the fast-ion temperature.

Velocity-space properties of the ambient fast-ion distribution function determine the resonance structure and the finite-orbit-width effects. In Maxwellian case, the fast-ion temperature is the only quantity entering explicitly the velocity part of the distribution function. However, there are also other means to affect the location of the resonances. The resonance condition for the fast-ion interaction with the wave can schematically be written as [7]:

$$
\omega-(m+\mu) \omega_{\theta}+\left(n+\nu N_{\text {per }}\right) \omega_{\varphi}=0
$$

Here, $\omega$ is the frequency, $m$ and $n$ are poloidal and toroidal numbers (respectively) of the wave, $\mu$ and $\nu$ describe the 3D geometry-induced coupling, $\omega_{\theta}$ and $\omega_{\varphi}$ are the frequencies of the poloidal and toroidal unperturbed motion of a fast ion. Now, these frequencies (especially the poloidal one) can be affected by the ambient radial electric field [see Eq. (3); here the ambient electric field enters through its potential $\left.\Phi_{0}\right]$. Hence, the resonant structure may be sensitive with respect to the ambient radial electric field since $\omega_{\theta} \sim E_{r}$. Indeed, our simulations reveal such a dependence. In Fig. 6, the mode growth rate is shown as a function of the Mach number $M_{E}=u_{E} / c_{s}$. Here, $u_{E}$ is the ambient $\mathbf{E} \times \mathbf{B}$ velocity computed at $s=0.5$ (employing a flat profile of the radial electric field) and $c_{s}=\sqrt{T_{e} / m_{i}}$ is the sound speed. One observes a gradual decrease of the mode growth rate when moving from the "ion root" (negative $E_{r}$ ) to the "electron root" (positive $E_{r}$ ) regime. Such a dependence may result from a combined effect of the phase-space resonance shift caused by $E_{r}$ and the FOW effects which bound the mode growth rate at higher fast-ion energies (temperatures). Note that the effect of a Doppler shift caused by the ambient $\mathbf{E} \times \mathbf{B}$ rotation should be very small for the Mach numbers considered. One can estimate it as $\delta \omega / \omega_{A} \sim u_{E} / v_{A} \sim M_{E} \sqrt{\beta} \ll 1$. The dependence of the TAE growth rate on the ambient electric field observed may be of practical interest since the sign of $E_{r}$ (electron or ion root) depends on neoclassical properties of the plasma (collisionality etc.) and can be actively manipulated (e. g. employing various heating scenarios). Also, the relative direction of the $\mathbf{E} \times \mathbf{B}$ rotation and the precession 
of trapped fast ions depends on whether the magnetic geometry is such that the parallel adiabatic invariant $J$ increases or decreases with minor radius [31].

Next, we consider the effect of anisotropy in the fast-ion background distribution function. One example of such an anisotropic distribution function is a combination of an isotropic Maxwellian (the same as has been used above) and a beam distribution (defined by its amplitude $\alpha_{b}$, its direction $\chi_{0}$, and its width $\Delta_{b}$; all are constants in the real space):

$$
F_{M b}=F_{M}\left[1+\alpha_{b} f_{b}(\chi)\right], \quad f_{b}(\chi)=\exp \left\{-\left(\frac{\chi-\chi_{0}}{\Delta_{b}}\right)^{2}\right\}, \quad \chi=v_{\|} / v
$$

Note that Eq. (19) can give both the "beam-like distributions" when $\alpha_{b}>1$ and $\chi_{0}$ is finite and the "loss-cone distributions" when $\alpha_{b}<0$ and $\chi_{0}=0$. In stellarators, the loss cones can appear due to the radial drift motion of locally-reflected particles (collisionless escape of energetic ions). An example of a loss-cone distribution function is shown in Fig. 7. This type of distribution-function anisotropy can be destabilising, as apparent from Fig. 8. Here, the growth rate is shown as a function of the fast-ion temperature computed for a varying loss-cone "width". The destabilisation is caused by the distribution-function gradient in the pitch angle (which leads, effectively, to a bump-on-tail structure). However, there are also other factors which affect the mode stability. For example, the number of resonant particles and the fast-ion beta are modified by the loss cone (diminished by the particle escape). This leads to stabilisation when the loss cone becomes larger (see Fig. 8).

Finally, consider an anisotropic (two-temperature) Maxwellian distribution function.

$$
f_{0}\left(s, v_{\|}, v_{\perp}\right)=\left(\frac{m_{h}}{2 \pi}\right)^{3 / 2} \frac{n_{h}(s)}{T_{\perp}(s) T_{\|}^{1 / 2}(s)} \exp \left[-\frac{m_{h} v_{\perp}^{2}}{2 T_{\perp}(s)}-\frac{m_{h} v_{\|}^{2}}{2 T_{\|}(s)}\right]
$$

An example of such a distribution function is shown in Fig. 9. In Ref. [32], a similar distribution function has been used to model the ICRH-heated "minority" ions whose perpendicular temperature was determined by the ICRH power deposition profile $[32,33]$ :

$$
T_{\perp}(s)=T_{e}(1+3 \xi / 2), \quad \xi=\frac{P_{\mathrm{RF}}(s) \tau_{s}}{3 n_{h}(r) T_{e}} \gg 1, \quad \tau_{s}=\frac{3(2 \pi)^{3 / 2} \epsilon_{0}^{2} m_{h} T_{e}^{3 / 2}}{Z_{h}^{2} e^{4} m_{e}^{1 / 2} n_{e} \ln \Lambda}
$$

with $\tau_{s}$ the slowing-down time and $P_{\mathrm{RF}}$ the Radio-Frequency $(\mathrm{RF})$ power deposition profile which we choose according to the expression:

$$
P_{\mathrm{RF}}(s)=P_{0} \exp \left[-\frac{\left(s-s_{\mathrm{ICRH}}\right)^{2}}{2 \Delta_{\mathrm{ICRH}}^{2}}\right]
$$


For the parallel temperature, we choose the following definition:

$$
T_{\|}(s)=T_{e}+\alpha_{T}\left[T_{\perp}(s)-T_{e}\right], \alpha_{T}<1
$$

Here, $\alpha_{T}$ is an anisotropy parameter considered to be constant for simplicity.

Consider now the TAE mode interaction with such "minority-ion" distribution functions. The minority-ion density is defined as in Sec. III A [see Eq. 15] with the same parameters $\left(N_{0}=10^{17} \mathrm{~m}^{-3}\right.$ etc). The perpendicular temperature is determined by the RF power deposition profile Eq. (22) with the parameters $s_{\mathrm{ICRH}}=0.8, \Delta_{\mathrm{ICRH}}=0.1$, and $P_{0}$ chosen appropriately to obtain the maximum perpendicular temperature required (see below). For the parameters chosen, the TAE mode with $m=(6,7)$ and $n=-6$ becomes unstable. This mode is shown in Fig. 10 along with the minority-ion density and the perpendicular temperature profiles. In Fig. 11, the growth rate is plotted as a function of the maximum minority-ion perpendicular temperature [with $T_{\max }=T_{\perp}\left(s_{\mathrm{ICRH}}\right)$, see Eqs. $(22)$ and $\left.(21)\right]$ for the anisotropy parameter $\alpha_{T}=0.2$. One sees that the FOW effects do not have much influence on the TAE growth rate (but the FLR effects do). This is caused by a localised fast-ion temperature profile chosen for the "minority ions" whose characteristic width (see Fig. 10) eventually becomes comparable to the fast-ion drift-orbit width. Note that a rather strong $\mathrm{RF}$ drive (large perpendicular temperatures) is required for the mode to become unstable. This is caused by the anisotropy of the distribution function: most of the fast-ion energy is "perpendicular" whereas the resonant mode destabilisation is determined by the parallel fast-ion temperature. The mode growth rate decreases with the temperature anisotropy as shown in Fig. 12. Of course, the distribution function Eq. (20) used here represents a rather crude model for the actual ICRH-driven distribution function in stellarator geometry. This model may still capture certain features of the real distribution function (such as the temperature anisotropy) but it misses other important effects (effects of finite ion orbit width, variations of the minority-ion distribution function along the flux surface, etc.). A more exact and comprehensive modelling is needed for the ICRH-driven minority ions in W7-X geometry to assess the role of such distribution-function properties on the Alfvénic stability. This problem is, however, beyond the scope of the present work and should be addressed in future. Only then will a quantitative prediction of the ICRH effect on the Alfvénic stability become feasible in W7-X. 


\section{Stability of Alfvén Eigenmodes in HELIAS geometry}

Fast particle confinement issues arising from their interaction with Alfvén Eigenmodes will be of particular importance under anticipated reactor-relevant plasma conditions. Here, we consider this topic in the case of a HELIAS configuration (the HELIcal Advanced Stellarator concept), which has been proposed as a candidate for the future DEMO reactor [14]. It is an extrapolation from W7-X based on present day knowledge. The basic parameters of HELIAS geometry are: $B_{0}=4.81 \mathrm{~T}$, major radius $R_{0}=20.3 \mathrm{~m}$, and minor radius $r_{a}=1.93 \mathrm{~m}$. The safety factor profile and the Fourier spectrum of the ambient magnetic field coincide with that of W7-X. Hence, the structure of the shear Alfvén continuum will be the same as it is in W7-X, provided the bulk-plasma density profiles coincide.

We start our considerations using the model plasma similar to that of Sec. III B, only under reactor-relevant conditions. Specifically, we implement flat bulk-plasma density $n_{\text {bulk }}=10^{20} \mathrm{~m}^{-3}$, flat bulk-plasma temperature $T_{i}=T_{e}=15 \mathrm{keV}$, Maxwellian distribution for the fast ions $\left(\mathrm{He}^{4}\right)$, and flat fast-ion temperature. The fast-ion density profile is given by Eq. (15) with $N_{0}=10^{18} \mathrm{~m}^{-3}$. For such parameters, average $\beta_{\text {fast }} \sim \beta_{\text {bulk }} \sim 0.05$ (when $\mathrm{He}^{4}$ fast ions with $3.5 \mathrm{MeV}$ energy are considered). Note that the average values (order of magnitude) of the densities and temperatures chosen here are consistent with the values predicted by the transport modelling (see below) of HELIAS plasmas. However, the profiles (their shape) are chosen to coincide with the profiles used in the W7-X simulations above (Sec. III B). For these profiles, the shear Alfvén continuum (normalised to the Alfvén frequency) coincides with the continuum shown in Fig. 2. We consider the TAE mode with the toroidal mode number $n=-6$ and the dominant poloidal mode numbers $m=(6,7)$ (the same mode has already been extensively studied in the original W7-X geometry, see Sec. IIIB). The eigenmode found in HELIAS geometry with the reduced-MHD eigenvalue solver $[34,35]$ is shown in Fig. 13. The growth rate of the unstable TAE mode is plotted as a function of the fast-ion temperature in Fig. 14. One sees that the FLR/FOW effects (stabilising under W7-X conditions, cf. Fig. 4) will be weak in the reactor plasma since the ratio of the fast-ion orbit width to the system size will be much smaller in the HELIAS reactor compared to $\mathrm{W} 7-\mathrm{X}$.

Finally, let us consider stability of the HELIAS plasma with respect to Alfvén Eigenmodes implementing realistic profiles predicted by the transport modelling (details of the transport 
code are described in Refs. [36, 37]). The transport model has been chosen to be mainly neoclassical in the bulk plasma with large anomalous transport at the edge. The anomalous diffusivity scales as $P^{0.75} / n$ where $P$ is the total heating power and $n$ is the electron density. At a developed stage of burn, the resulting energy diffusivities at the plasma edge are between $1-5 \mathrm{~m}^{2} / \mathrm{s}$, while in the plasma core they are about $1 \mathrm{~m}^{2} / \mathrm{s}$ for electrons and $1.5 \mathrm{~m}^{2} / \mathrm{s}$ for deuterium ions. The particle source, used in the transport modelling, is shown in Fig. 15(a). The bulk-plasma densities, temperatures, production rate of fusion alphas, corresponding fast-ion density, the fast-ion and the bulk-plasma betas obtained in the modelling are shown in Figs. 15(b-e). Note that the projected steady-state fusion energy gain factor $Q_{\text {steady }}=\infty$ for the HELIAS reactor which requires higher pressure of the energetic alphas (compared to burning plasmas with smaller $\left.Q_{\text {steady }}\right)$. The shear Alfvén continuum corresponding to the predicted bulk-ion density profile is plotted in Fig. 16. Here, one sees that the largest gap in the continuum corresponds to the helical coupling of the Fourier harmonics ("helicityinduced gap"). The Helical Alfvén Eigenmode (HAE) with the dominant ( $m=-14, n=11)$ and $(m=-16, n=16)$ Fourier harmonics, which is located in this gap, is shown in Fig. 17. The steady-state distribution function of the energetic alpha particle is modelled with a slowing-down distribution function Eq. (16) corresponding to the plasma profiles predicted by the transport modelling. In the case considered, the HAE mode is unstable. The growth rate of the HAE, $\gamma=1.8 \times 10^{4} \mathrm{rad} / \mathrm{s}$, and the frequency $\omega=-4.1 \times 10^{5} \mathrm{rad} / \mathrm{s}$, have the ratio $\gamma / \omega \sim 4.4 \%$.

The unstable Alfvén Eigenmodes may cause fast-ion transport (in nonlinear regime). The nonlinear fluctuation channel could couple to the usual collisionless "3D-geometry" channel (toroidal magnetic-field ripple loss). Such a synergy between different types of fastion transport (AE-induced ripple trapping [38]) may become an issue in burning stellarator plasmas and deserves further consideration.

\section{CONCLUSION}

In this paper, we have studied the interplay of energetic ions and Alfvén eigenmodes in stellarator plasmas. The Wendelstein 7-X stellarator and its extrapolation to a reactor-scale HELIAS configuration have been considered. A hybrid reduced-MHD gyrokinetic numerical framework has been used in order to study AE mode stability in these plasmas. FOW and 
FLR stabilisation effects have been observed in the W7-X plasma, but are much weaker in the reactor. Furthermore, an effect of the equilibrium radial electric field (stabilising in the electron root) has been demonstrated. This effect may be attributed to the modification of the drift fast-ion orbits in presence of the electric field. An anisotropy in the background fast-ion distribution function has been considered in the cases of a "loss-cone" and an anisotropic two-temperature Maxwellian distribution functions. The two-temperature Maxwellian anisotropy may inhibit AE mode destabilisation since in this case most of the fast-ion energy is concentrated in the perpendicular particle motion. In the reactor plasma, the stability properties have been considered under conditions predicted by the transport modelling. An unstable HAE mode has been found with $\gamma / \omega \approx 4 \%$.

Of course, it must be borne in mind that we have only calculated the drive and damping directly related to the fast ions. All the damping mechanisms associated with the bulk plasma (collisional, continuum and radiative damping) have been ignored. Nevertheless, the calculation shows that AEs could be driven unstable by alpha particles in a stellarator reactor. A careful evaluation of the damping is thus called for. In this respect, a stepwise approach is envisioned. As a first step, a fluid-electron gyrokinetic-ion model will be employed to the cases already considered with the perturbative hybrid approach presented in this paper. This model, still reduced, can however describe at a sufficient level of accuracy interaction of AEs with shear Alfvén continuum in a non-perturbative fashion. Such an interaction is considered to be responsible for the continuum and radiative damping mechanisms (see e. g. Refs. [39-41]). The fluid-electron gyrokinetic-ion model is already under development and will be described in a separate publication. More comprehensive but also rather expensive (computationally) full-gyrokinetic simulations will be undertaken after the fluid-electron results become feasible. Similar simulations have already been carried out in tokamak geometry [30, 42, 43]. Furthermore, realistic simulations of NBI- and ICRH-heated W7-X plasmas using real (predicted) anisotropic background distribution functions as well as predicted plasma profiles should be undertaken using the perturbative hybrid-gyrokinetic approach. Such simulations would be of interest as a preparation for experimental work on W7-X. Finally, a perturbative modelling, being technically very robust, has the drawback of working with preselected eigenmodes which, however, do not need to be dominant in the actual stability. Thus, a comprehensive assessment of Alfvén modes in stellarators will require a non-perturbative framework (such as the aforementioned fluid-electron gyrokinetic-ion 
model or full-gyrokinetic approach). The work on the non-perturbative schemes is ongoing and will be reported elsewhere.

\section{ACKNOWLEDGMENTS}

We acknowledge P. Helander who has supported this work. The simulations have been performed on the HPC-FF supercomputer (Jülich Supercomputing Centre, Germany) as well as on the local cluster in Greifswald (H. Leyh is appreciated). We acknowledge fruitful discussions with N. Marushchenko.

[1] W. W. Heidbrink, Phys. Plasmas 15, 055501 (2008).

[2] K. Toi, M. Takechi, M. Isobe, N. Nakajima, M. Osakabe, S. Takagi, T. Kondo, G. Matsunaga, K. Ohkuni, M. Sasao, S. Yamamoto, S. Ohdachi, S. Sakakibara, H. Yamada, K. Y. Watanabe, D. S. Darrow, A. Fujisawa, M. Goto, K. Ida, H. Idei, H. Iguchi, S. Lee, S. Kado, S. Kubo, O. Kaneko, K. Kawahata, K. Matsuoka, T. Minami, S. Morita, O. Motojima, K. Narihara, S. Nishimura, N. Ohyabu, Y. Oka, S. Okamura, T. Ozaki, K. Sato, M. Sato, A. Shimizu, T. Shimozuma, Y. Takeiri, K. Tanaka, T. Tokuzawa, K. Tsumori, I. Yamada, Y. Yoshimura and CHS and LHD Experimental Groups, Nucl. Fusion 40, 1349 (2000).

[3] A. Weller, M. Anton, J. Geiger, M. Hirsch, R. Jaenicke, A. Werner, C. Nührenberg, E. Sallander, and D. A. Spong, Phys. Plasmas 8, 931 (2001).

[4] F. Zonca and L. Chen, Plasma Phys. Controlled Fusion 48, 537 (2006).

[5] F. Zonca, S. Briguglio, L. Chen, C. Fogaccia, T. S. Hahm, A. V. Milovanov, and G. Vlad, Plasma Phys. Controlled Fusion 48, B15 (2006).

[6] B. Breizman and S. Sharapov, Plasma Phys. Controlled Fusion 53, 054001 (2011).

[7] Ya. I. Kolesnichenko, A. Könies, V. V. Lutsenko, and Yu. V. Yakovenko, Plasma Phys. Controlled Fusion 53, 024007 (2011).

[8] Ya. I. Kolesnichenko, V. V. Lutsenko, H. Wobig, Yu. V. Yakovenko, and O. P. Fesenyuk, Phys. Plasmas 8, 491 (2001).

[9] Ya. I. Kolesnichenko, V. V. Lutsenko, H. Wobig, and Yu. V. Yakovenko, Phys. Plasmas 9, 517 
(2002).

[10] H. E. Mynick, Phys. Plasmas 13, 058102 (2006).

[11] G. Grieger, W. Lotz, P. Merkel, J. Nührenberg, J. Sapper, E. Strumberger, H. Wobig and the W7-X Team, R. Burhen, V. Erckmann, U. Gasparino, L. Giannone, H. J. Hartfuss, R. Jaenicke, G. Kühner, H. Ringler, A. Weller, F. Wagner and the W7-AS-Team, Phys. Fluids 4, 2081 (1992).

[12] O. Motojima, N. Ohyabu, A. Komori, O. Kaneko, S. Masuzaki, A. Ejiri, M. Emoto, H. Funaba, M. Goto, K. Ida, H. Idei, S. Inagaki, N. Inoue, S. Kado, S. Kubo, R. Kumazawa, T. Minami, J. Miyazawa, T. Morisaki, S. Morita, S. Murakami, S. Muto, T. Mutoh, Y. Nagayama, Y. Nakamura, H. Nakanishi, K. Narihara, K. Nishimura, N. Noda, T. Kobuchi, S. Ohdachi, Y. Oka, M. Osakabe, T. Ozaki, B. J. Peterson, A. Sagara, S. Sakakibara, R. Sakamoto, H. Sasao, M. Sasao, K. Sato, M. Sato, T. Seki, T. Shimozuma, M. Shoji, H. Suzuki, Y. Takeiri, K. Tanaka, K. Toi, T. Tokuzawa, K. Tsumori, K. Tsuzuki, I. Yamada, S. Yamaguchi, M. Yokoyama, K. Y. Watanabe, T. Watari, Y. Hamada, K. Matsuoka, K. Murai, K. Ohkubo, I. Ohtake, M. Okamoto, S. Satoh, T. Satow, S. Sudo, S. Tanahashi, K. Yamazaki, M. Fujiwara, and A. Iiyoshi, Nucl. Fusion 43, 1674 (2003).

[13] J. Kisslinger, C. D. Beidler, and E. Strumberger, in Controlled Fusion and Plasma Physics, Abstracts of Invited and Contributed Papers, 27th European Physical Society Conference, Budapest (The European Physical Society, Petit-Lancy, 2000, 2000), Vol. 1069.

[14] R. C. Wolf, C. D. Beidler, S. Bozhenkov, V. Bykov, A. Dinklage, K. Egorov, Y. Feng, P. Helander, H. Maassberg, F. Schauer, Y. Turkin, F. Warmer, P. Xanthopoulos, H. Zohm and the Wendelstein 7-X Team, in 1st IAEA DEMO Programme Workshop: Power plant studies based on the HELIAS Stellarator Line (IAEA, Vienna, Austria, 2012).

[15] Ya. I. Kolesnichenko, S. Yamamoto, K. Yamazaki, V. V. Lutsenko, N. Nakajima, Y. Narushima, K. Toi, and Yu. V. Yakovenko, Phys. Plasmas 11, 158 (2004).

[16] V. S. Marchenko, Phys. Plasmas 15, 102504 (2008).

[17] V. Kornilov, R. Kleiber, R. Hatzky, L. Villard, and G. Jost, Phys. Plasmas 11, 3196 (2004).

[18] T. Feher, Ph.D. thesis, Max-Planck-Institut für Plasmaphysik, Greifswald, 2013.

[19] A. Könies, in IAEA TM on Energetic Particles (IAEA, Kloster Seon, 2007).

[20] S. P. Hirshman, W. I. van Rij, and P. Merkel, Comp. Phys. Commun. 43, 143 (1986).

[21] G. Grieger, C. D. Beidler, H. Maassberg, E. Harmeyer, F. Herrnegger, J. Junker, J. Kisslinger, 
W. Lotz, P. Merkel, J. Nührenberg, F. Rau, J. Sapper, A. Schlüter, F. Sardei, and H. Wobig, in Proc. of the 13th International Conference on Plasma Physics and Controlled Nuclear Fusion Research (International Atomic Energy Agency, Vienna, 1991), Vol. 3, p. 525.

[22] W. Lotz, J. Nührenberg, and C. Schwab, in Proceedings of the 13th International Conference on Plasma Physics and Controlled Nuclear Fusion Research (Washington, DC, 1990) (International Atomic Energy Agency, Vienna, 1991), Vol. 3, p. 603.

[23] T. S. Hahm, W. W. Lee, and A. J. Brizard, Phys. Fluids 31, 1940 (1988).

[24] P. Helander and A. Simakov, Phys. Rev. Lett. 101, 145003 (2008).

[25] W. W. Lee, J. Comp. Phys. 72, 243 (1987).

[26] A. Mishchenko, A. Könies, and R. Hatzky, Phys. Plasmas 12, 062305 (2005).

[27] C. de Boor, A Practical Guide to Splines (Springer-Verlag, New York, 1978).

[28] K. Höllig, Finite Element Methods with B-Splines (Society for Industrial and Applied Mathematics, Philadelphia, 2003).

[29] C. Schwab, Phys. Fluids B 5, 3195 (1993).

[30] A. Mishchenko, A. Könies, and R. Hatzky, Phys. Plasmas 16, 082105 (2009).

[31] P. Helander, C. D. Beidler, T. M. Bird, M. Drevlak, Y. Feng, R. Hatzky, F. Jenko, R. Kleiber, J. H. E. Proll, Yu. Turkin, and P. Xanthopoulos, Plasma Phys. Controlled Fusion 54, 124009 (2012).

[32] R. O. Dendy, R. J. Hastie, K. G. McClements, and T. J. Martin, Phys. Plasmas 2, 1623 (1995).

[33] T. H. Stix, Nucl. Fusion 15, 737 (1975).

[34] A. Könies, Phys. Plasmas 7, 1139 (2000).

[35] A. Könies, A. Mishchenko, and R. Hatzky, in Proc. of the Joint Varenna-Lausanne International Workshop (AIP Conference Proceedings, New York, 2008), Vol. 1069.

[36] Yu. Turkin, H. Maassberg, C. D. Beidler, J. Geiger, and N. B. Marushchenko, Fusion Sci. Technol. 50, 387 (2006).

[37] Yu. Turkin, C. D. Beidler, H. Maassberg, S. Murakami, V. Tribaldos, and A. Wakasa, Phys. Plasmas 18, 022505 (2010).

[38] R. B. White, Y. Wu, Y. Chen, E. D. Fredrickson, D. S. Darrow, M. C. Zarnstorff, J. R. Wilson, S. J. Zweben, K. W. Hill, and G. Y. Fu, Nucl. Fusion 35, 1707 (1995).

[39] F. Zonca and L. Chen, Phys. Rev. Lett. 68, 592 (1992). 
[40] H. L. Berk, J. W. Van Dam, Z. Guo, and D. M. Lindberg, Phys. Fluids B 4, 1806 (1992).

[41] M. N. Rosenbluth, H. L. Berk, J. W. Van Dam, and D. M. Lindberg, Phys. Fluids B 4, 2189 (1992).

[42] A. Mishchenko, R. Hatzky, and A. Könies, Phys. Plasmas 15, 112106 (2008).

[43] A. Mishchenko, A. Könies, and R. Hatzky, Phys. Plasmas 18, 012504 (2011). 


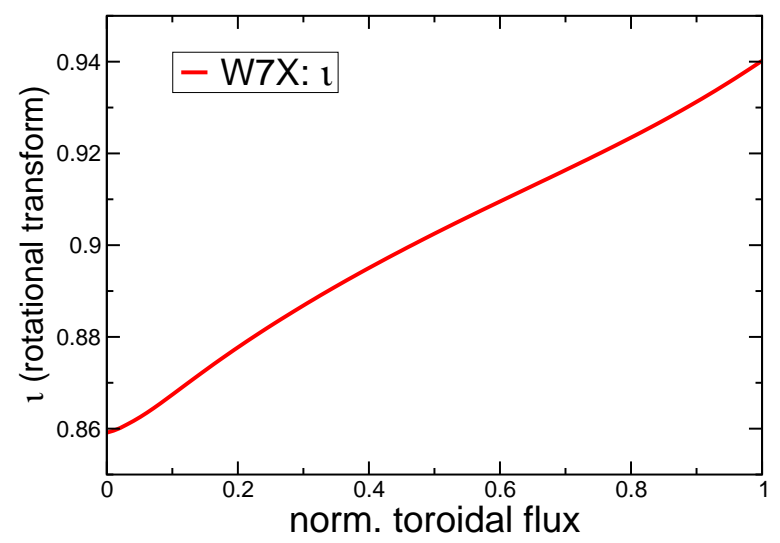

FIG. 1: (Colour online) Rotational transform in W7-X (high-mirror configuration). 


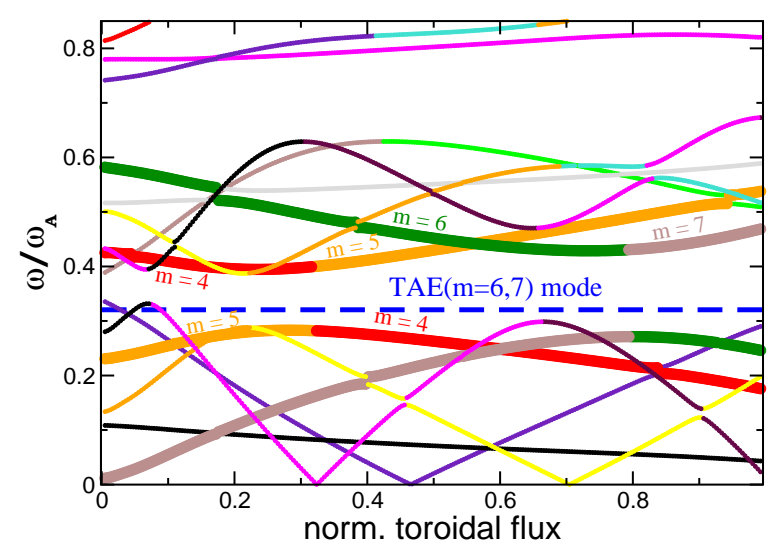

FIG. 2: (Colour online) Shear Alfvén wave continuum in the W7-X configuration $(n=1$ mode family) corresponding to flat bulk plasma density $n_{\text {bulk }}=2 \times 10^{20} \mathrm{~m}^{-3}$. One can see the toroidicityinduced gap in the spectrum. The TAE eigenmode frequency (blue straight line) corresponding to the toroidal mode number $n=-6$ and the coupled poloidal mode numbers $m=6$ (green curve) and $m=7$ (brown curve) is shown inside the gap. Here, the Alfvén frequency $\omega_{A}=7.4 \times 10^{5} \mathrm{rad} / \mathrm{s}$. 


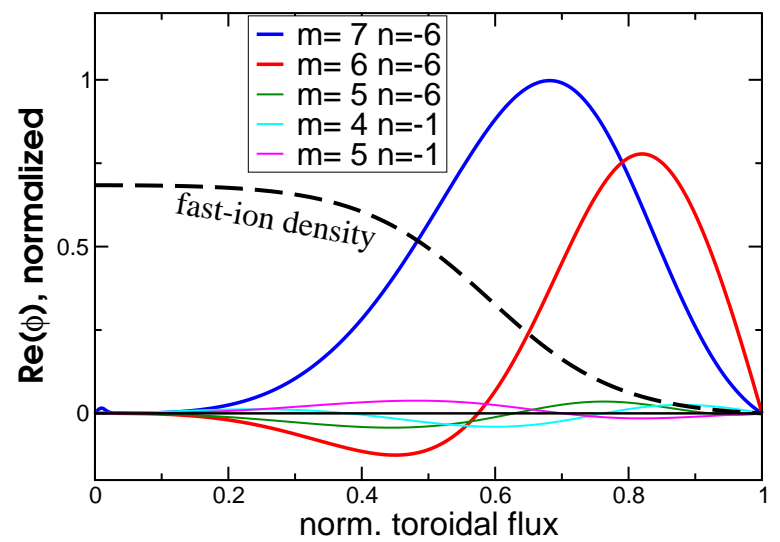

FIG. 3: (Colour online) The eigenfunction corresponding to the global (even) TAE mode (see the eigenfrequency in Fig. 2). One sees that $m=6$ and $m=7$ poloidal harmonics are coupled (and dominant), in accordance with the shear Alfvén spectrum shown in Fig. 2. The maximum of the mode is located near $s=0.65$ (at the position of the TAE accumulation point). 


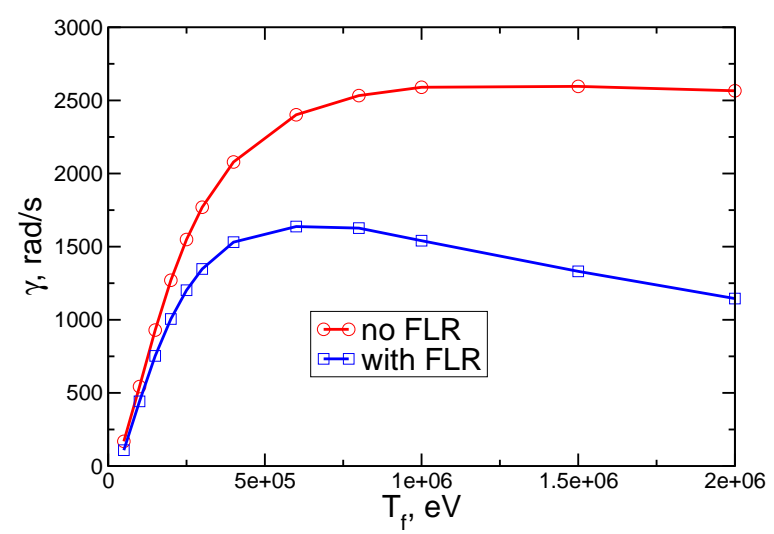

FIG. 4: (Colour online) Growth rate of the TAE mode (W7-X geometry) as a function of the fastparticle temperature at fixed fast-ion density $N_{0}=10^{17} \mathrm{~m}^{-3}$ (Maxwellian fast-particle distribution has been used). The growth rates without FLR effects (drift-kinetic fast ions) and with FLR effects (gyrokinetic fast ions) have been considered. The frequency of the TAE mode $\omega_{\mathrm{TAE}}=238766 \mathrm{rad} / \mathrm{s}$. The fast-ion beta range (measured at the position of maximal fast-ion density gradient $s=0.65$ ) is $0.0006 \leq \beta_{f} \leq 0.012$. For the bulk plasma, $\beta_{\text {bulk }}(s=0.65)=0.034$. 


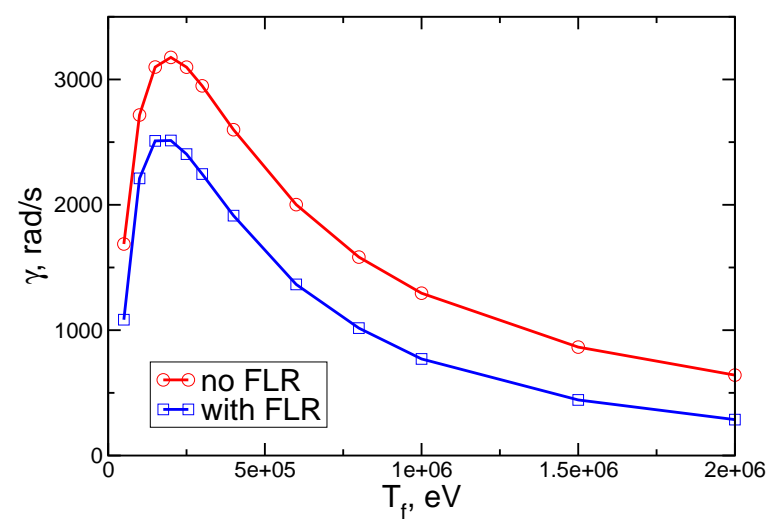

FIG. 5: (Colour online) Growth rate of the TAE mode (W7-X geometry) as a function of the fast-particle temperature at fixed fast-ion beta $\beta_{f}(s=0.65) \approx 0.003$. Here, bulk-plasma beta $\beta_{\text {bulk }}(s=0.65)=0.034$ and other parameters are the same as in Fig. 4. 


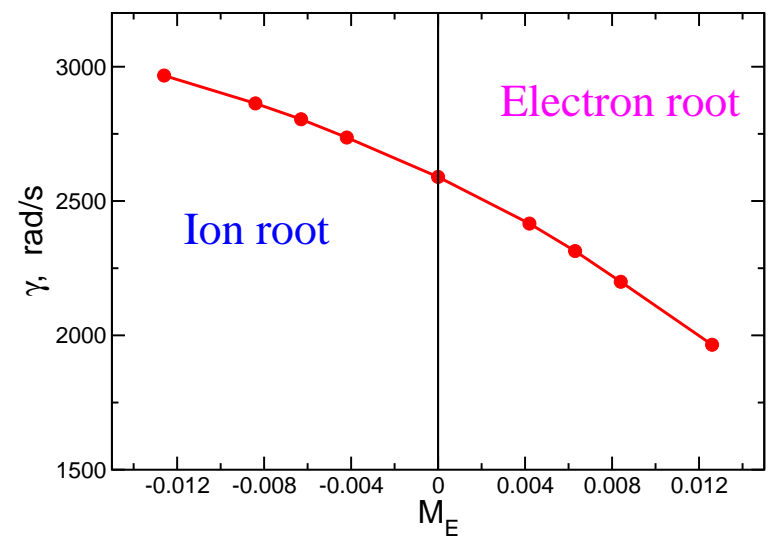

FIG. 6: (Colour online) Growth rate as a function of the ambient radial electric field (FLR effects neglected). Here, the fast-particle temperature $T_{f}=1 \mathrm{MeV}$. 


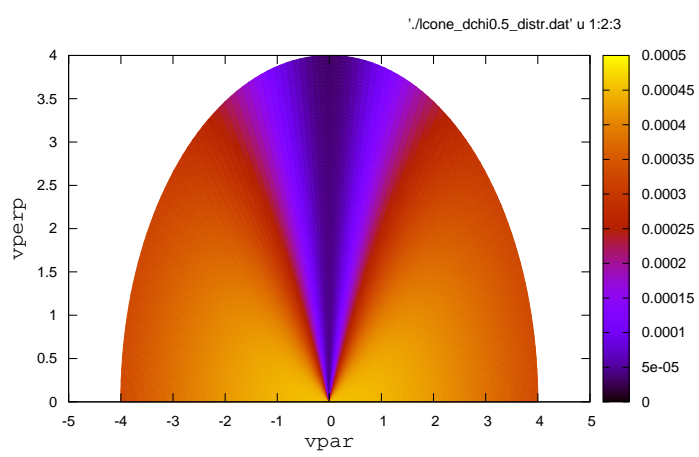

FIG. 7: (Colour online) Loss-cone distribution function with parameters $\chi_{0}=0, \Delta_{b}=0.5$, and $\alpha_{b}=-0.9$ projected onto the $\left(v_{\|}, v_{\perp}\right)$-plane (here $v_{\|}$corresponds to the horizontal axis and $v_{\perp}$ to the vertical axis). 


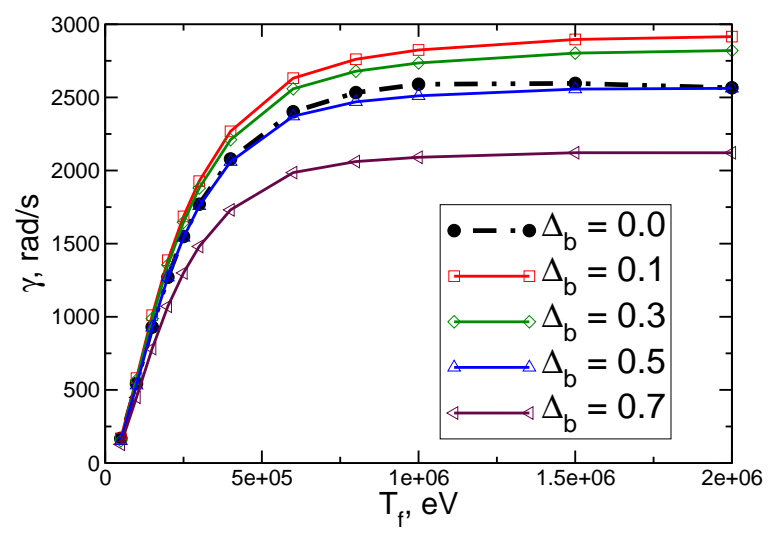

FIG. 8: (Colour online) Growth rate of the TAE mode (W7-X geometry) as a function of the fast-particle temperature in presence of a loss cone in the distribution function. The growth rates are plotted at different "widths of the loss cone". One sees that the dependence of the TAEmode growth rate on the loss-cone width is non-monotonic: there is a competition between the anisotropy drive (which wins at smaller "loss cones") and stabilisation caused by decreasing fastparticle pressure (caused by the "prompt losses" and winning when $\Delta_{b}$ increases). Here, $\alpha_{b}=-0.9$ [see Eq. (19)]. 


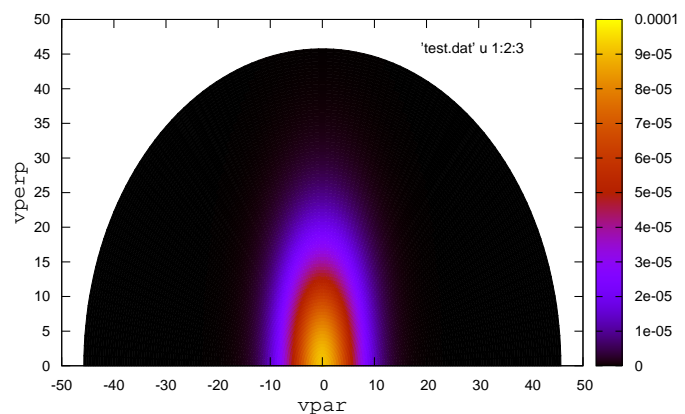

FIG. 9: (Colour online) ICRH-type distribution function $f_{0}\left(v_{\|}, v_{\perp}\right)$ shown as a function of $v_{\|}$ (horizontal axis) and $v_{\perp}$ (vertical axis). 


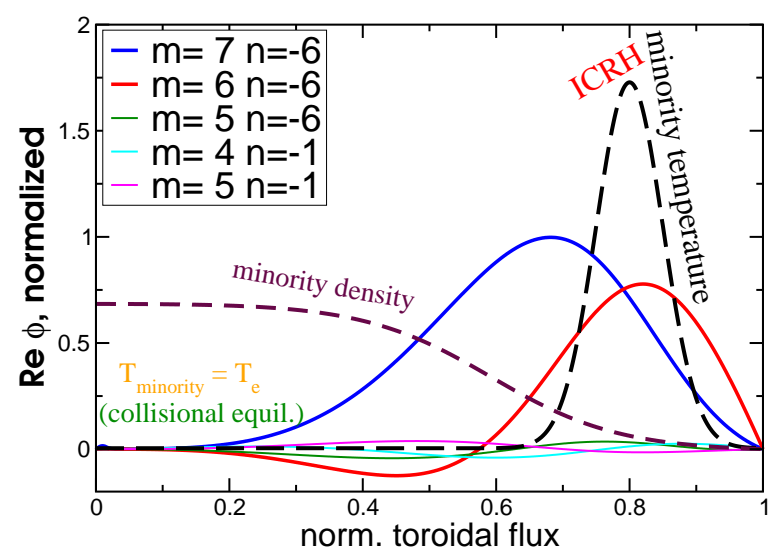

FIG. 10: (Colour online) Unstable TAE eigenfunction and plasma profiles (used as a proxy for the ICRH scenario). 


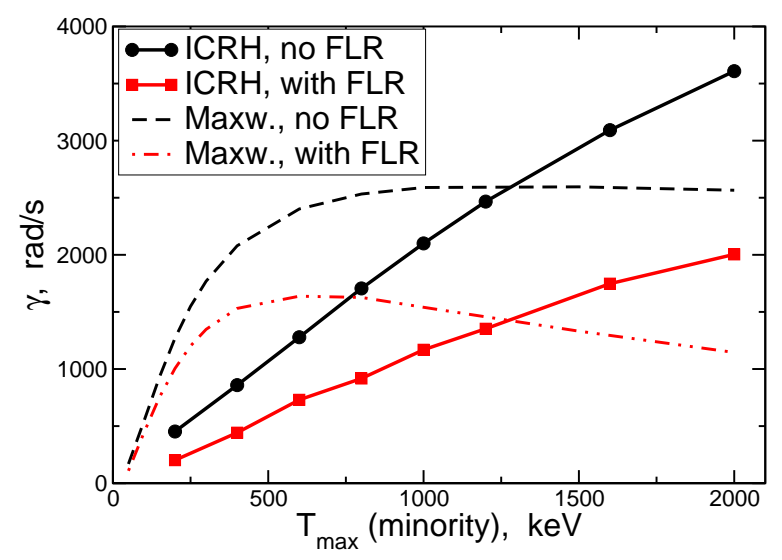

FIG. 11: (Colour online) Growth rate as a function of the maximal minority-ion perpendicular temperature $T_{\max }$ (related to the RF power) in the ICRH-type scenario. The anisotropic Maxwellian is compared with the isotropic one (defined using the same density profile and a flat temperature equal to the ICRH maximum $T_{\perp}$ ). The stabilising FOW effect is weak in the anisotropic case, which is probably due to the strong localisation of the energetic-ion temperature profile. Note that $T_{\max }=400 \mathrm{keV}$ corresponds roughly to the maximum ICRH power $P_{0}=3 \mathrm{MW} / \mathrm{m}^{3}$. 


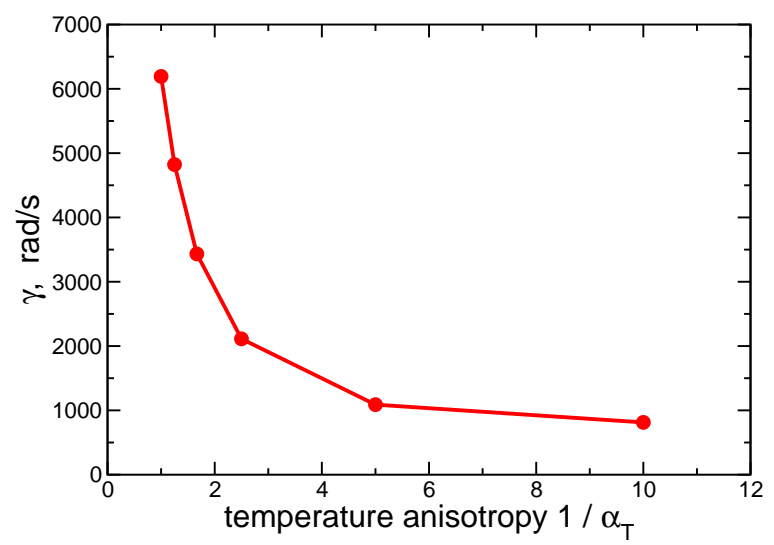

FIG. 12: (Colour online) Effect of the temperature anisotropy. The parameter $\alpha_{T}$ defines the ratio of the parallel temperature to the perpendicular one. Here, the maximum perpendicular temperature ("ICRH-driven tail" in the distribution function) was $T_{\perp}=400 \mathrm{keV}$. Note that the isotropic case $\alpha_{T}=1$ is more unstable for the inhomogeneous minority-ion temperature profile used here (see Fig. 10) compared to the Maxwellian with the same density but flat temperature profile (Fig. 4). 


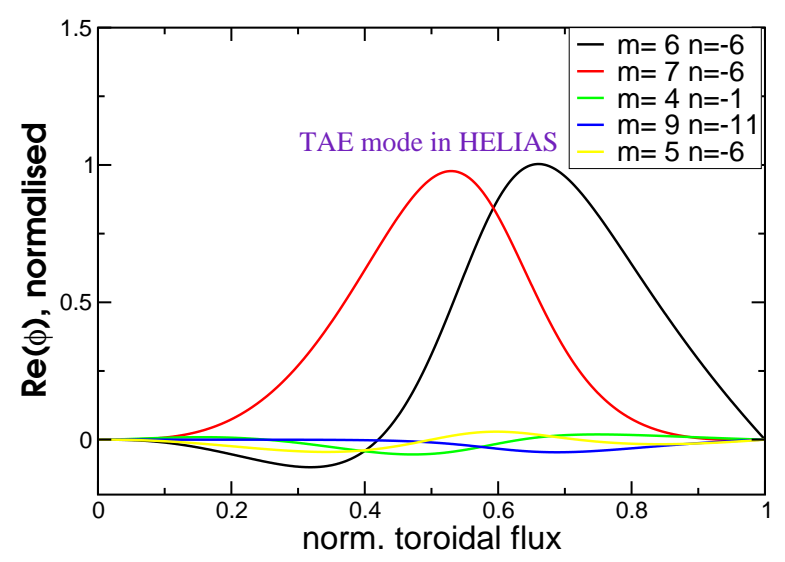

FIG. 13: (Colour online) Unstable TAE in HELIAS geometry (assuming flat bulk-plasma density). The frequency of the mode $\omega=111796 \mathrm{rad} / \mathrm{s}$. 


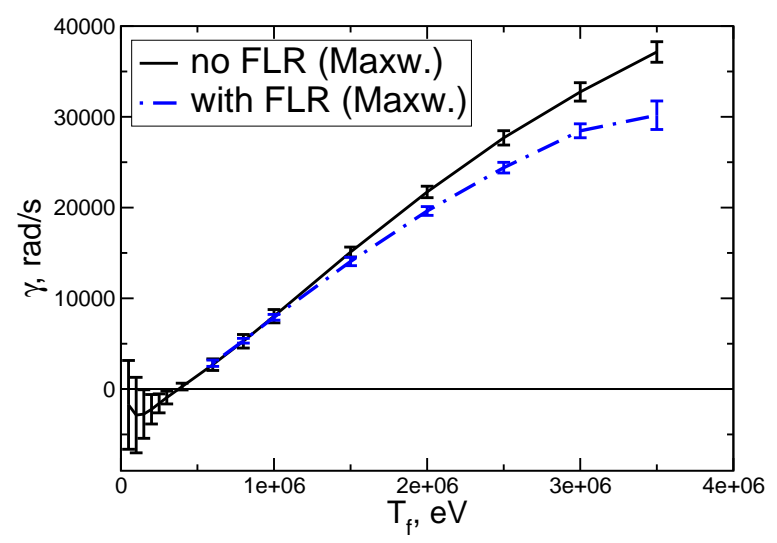

FIG. 14: (Colour online) TAE growth rate in the HELIAS reactor as a function of fast-ion temperature. It is striking how little the FLR/FOW stabilisation mechanisms matter in the reactor environment. The frequency of the mode $\omega=111796 \mathrm{rad} / \mathrm{s}$. 

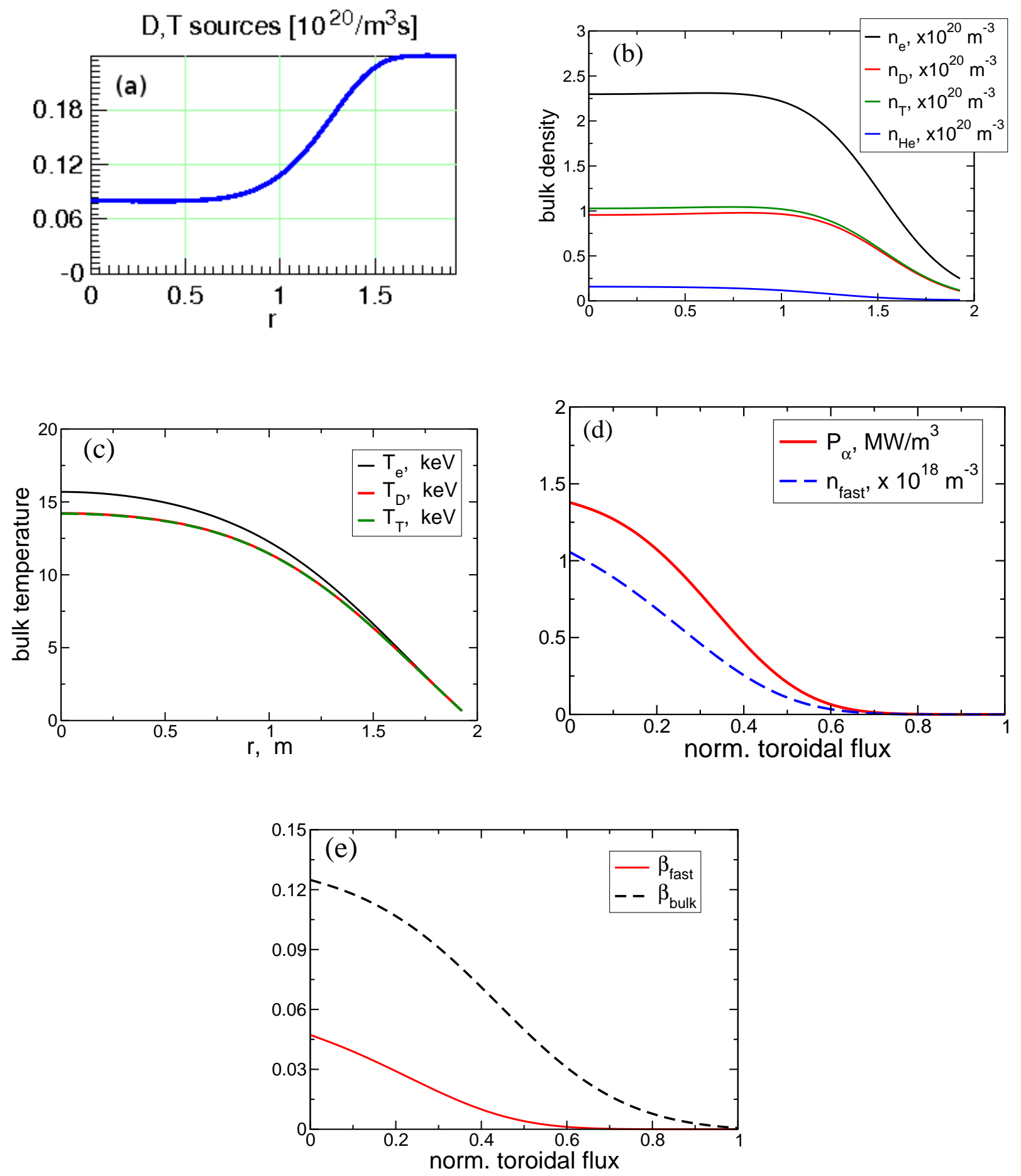

FIG. 15: (Colour online) (a) Profile of the particle (D-T) sources, used in the transport modelling of the HELIAS plasma, plotted as a function of $r=r_{a} \sqrt{s}$ where $s$ is the normalised toroidal flux and $r_{a}$ is the minor radius of the device. (b) Predicted plasma density profiles (transport calculations): electron, deuterium, tritium, and helium-ash densities. (c) Predicted plasma temperature profiles (transport calculations). (d) Predicted power density of fusion alphas and the resulting energeticion density (computed as $n_{\text {fast }}=\int F_{\text {sd }} \mathrm{d}^{3} v$ ). (e) Predicted fast-ion and bulk-ion betas. The fast-ion beta $\beta_{\text {fast }}=2 \mu_{0} p_{\text {fast }} / B^{2}$ with the fast-ion pressure roughly estimated as $p_{\text {fast }} \approx P_{\alpha} \tau_{s}$. 


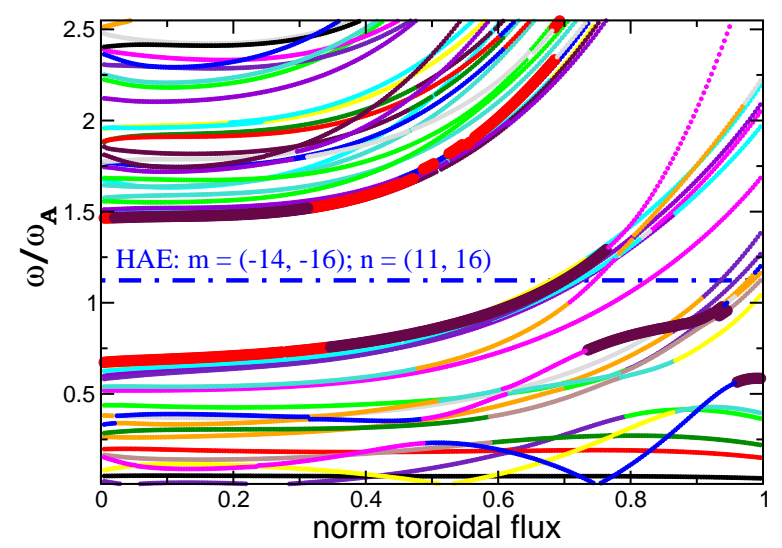

FIG. 16: (Colour online) Shear Alfvén continuum generated by the predicted bulk plasma density profile in HELIAS geometry. The frequency of the HAE mode with the dominant $(m=-14, n=$ 11) and $(m=-16, n=16)$ Fourier harmonics is also plotted. Here, the Alfvén frequency $\omega_{A}=365453 \mathrm{rad} / \mathrm{s}$. 


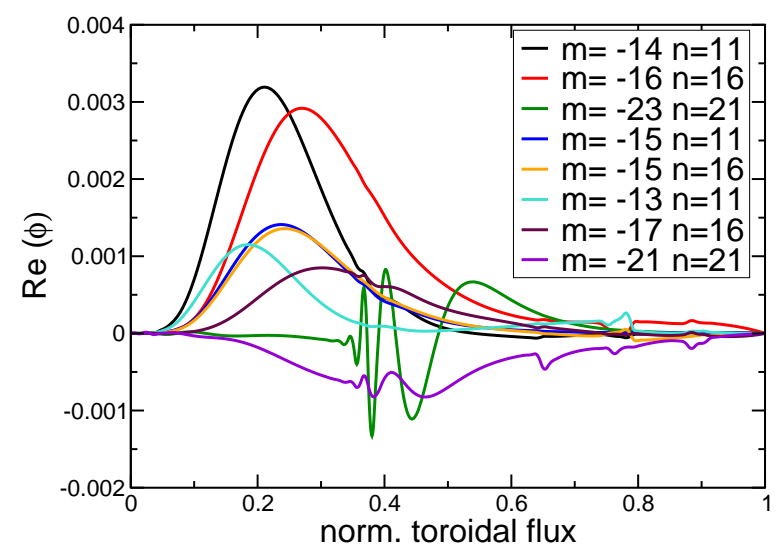

FIG. 17: (Colour online) The HAE mode is unstable for the predicted profiles. The growth rate of this mode $\gamma=17874 \mathrm{rad} / \mathrm{s}$, and the frequency $\omega=-410523.29 \mathrm{rad} / \mathrm{s}$. 